\title{
Los colores del tejido adiposo y la relación con la irisina
}

\section{The colors of adipose tissue and the relationship with irisin}

\author{
Betsy Corina-Sosa',2, Lourdes Basurto ${ }^{*}$, Eva Luqueño ${ }^{1,3}$, Ariadna Robledo ${ }^{1}$, Hugo Mendieta-Zerón² y \\ Rigoberto Oros-Pantoja² \\ ${ }^{1}$ Unidad de Investigación Médica en Enfermedades Endocrinas, Centro Médico Nacional, Instituto Mexicano del Seguro Social, Ciudad de México; \\ ${ }^{2}$ Facultad de Medicina, Universidad Autónoma del Estado de México, Toluca, Estado de México; ${ }^{3}$ Facultad de Medicina, Universidad Autónoma \\ del Estado de Hidalgo, Pachuca, Hidalgo. México
}

\begin{abstract}
Resumen
El exceso de tejido adiposo representa una de las principales causas de las enfermedades metabólicas y cardiovasculares. Inicialmente al tejido adiposo se le consideró el almacén de lípidos y energía del organismo. Posteriormente se descubrió que presentaba otras funciones, como la endocrina, controlando diferentes procesos metabólicos e inmunitarios. Por sus características funcionales y estructurales, se reconocen varios tipos de tejido adiposo. El adipocito blanco representa la reserva energética y el adipocito marrón se encarga de la oxidación de los lípidos para la termogénesis. El adipocito beige se origina del adipocito blanco, mediante un proceso que conduce a la lipólisis y la termogénesis. A los anteriores se han sumado el adipocito azul, en el hígado, que interviene en la homeostasis de retinoides, y el adipocito rosa, que participa en la lactogénesis y se ha identificado en la glándula mamaria de animales. La irisina es una hormona secretada principalmente por el músculo esquelético, que promueve el pardeamiento del tejido adiposo blanco a beige, favoreciendo así la termogénesis. Otro aspecto interesante de esta hormona es que representa una conexión entre la actividad muscular y la lipólisis. Por lo anterior, la irisina puede ser una clave en la prevención y el tratamiento de la obesidad.
\end{abstract}

Palabras clave: Adipocito azul. Adipocito beige. Adipocito blanco. Adipocito marrón. Adipocito rosa. Irisina.

\begin{abstract}
Excess adipose tissue is considered one of the main causes of metabolic and cardiovascular diseases. Initially, the adipose tissue was considered the main lipid and energy storage of the organism. Subsequently it was discovered that adipose tissue had other functions such as endocrine, controlling different metabolic and immune processes. Currently, different types of adipose tissue are recognized. The white adipocyte represents the main energy reserve, on the contrary the brown adipocyte is responsible for the oxidation of lipids for thermogenesis. The beige adipocyte originates from the white adipocyte, by a process known as "browning", which leads to lipolysis and thermogenesis. The 3 previous types have recently joined the blue adipocyte, which has a role in liver retinoid homeostasis and the pink adipocyte that participates in lactogenesis and is present in the mammary gland of animals; its role is still unknown in humans. The newly identified hormone Irisin is secreted by the skeletal muscle and promotes browning of white to beige adipose tissue, thus favoring thermogenesis. Another interesting aspect of this hormone is that it represents a connection between muscle activity and lipolysis. The above suggests that Irisin may be the key in the prevention and treatment of obesity.
\end{abstract}

Key words: Blue adipocyte. Beige adipocyte. White adipocyte. Brown adipocyte. Pink adipocyte. Irisin.

\section{Correspondencia:}

*Lourdes Basurto-Acevedo

Avda. Cuauhtémoc, 330

Col. Doctores, Del. Cuauhtémoc

Fecha de recepción:30-01-2020

C.P. 06720, Ciudad de México, México

E-mail: Ibasurtoa@yahoo.com
Cir Cir. 2020;88(5):664-671

Contents available at PubMed www.cirugiaycirujanos.com (http://creativecommons.org/licenses/by-nc-nd/4.0/) 


\section{Introducción}

El tejido adiposo, que por mucho tiempo fue considerado únicamente como un almacén de energía, cumple con otras funciones, como la endocrina, al sintetizar moléculas que controlan diversas acciones relacionadas con el metabolismo de la glucosa, los lípidos y la sensibilidad a la insulina'.

El tejido adiposo se encuentra distribuido en distintas regiones del organismo, por lo que puede ser subcutáneo (región cervical, craneal, facial, supraclavicular, glúteo y abdominal), retroorbital, paravertebral, epicárdico, omental, retroperitoneal, visceral y perigonadal ${ }^{2}$. Se ha observado que la localización del tejido adiposo determina la presencia de notables diferencias en las características y las funciones de los distintos tipos de adipocitos. En particular, el tejido adiposo visceral ha cobrado relevancia por su asociación con el desarrollo de la aterosclerosis, la regulación metabólica, la hipertensión arterial y la dislipidemia ${ }^{3}$.

El tejido adiposo epicárdico también ha despertado interés por ser metabólicamente activo y por su relación con la aterosclerosis coronaria ${ }^{4,5}$. Se ha propuesto que la grasa epicárdica es un reservorio energético para el miocardio, y por su actividad metabólica influye también en la producción de numerosas adipocinas, incluyendo citocinas proinflamatorias $y$ proaterogénicas como el factor de necrosis tumoral alfa (TNF- $\alpha$ ), el inhibidor del activador del plasminógeno tipo 1, la interleucina 6, la visfatina, la leptina, la omentina y la angiotensina ${ }^{6}$.

\section{Los colores del adipocito}

En la clasificación del tejido adiposo según su color se consideraron originalmente el tejido blanco (WAT, white adipose tissue) y el tejido marrón o pardo (BAT, brown adipose tissue). Posteriormente se identificó el tejido adiposo beige y recientemente se han descrito dos tipos diferentes de adipocitos, que por sus características histológicas reciben el nombre de adipocito rosa y adipocito azul ${ }^{7,8}$.

Se puede decir de manera sencilla que el adipocito blanco almacena lípidos y que el marrón produce calor. Ambos tipos de adipocitos comparten una serie de genes y enzimas relacionados con el metabolismo de los triglicéridos, y por largo tiempo se consideró que presentaban un desarrollo común. Hace algunos años, los estudios mostraron que los adipocitos blancos y marrones no provenían en su desarrollo de la misma célula original. El adipocito blanco tiene su origen en el adipoblasto. El adipocito marrón, por su parte, tiene más cercanía con las células del músculo esquelético, con las que comparte un mismo precursor (Fig. 1). El Myf5 (myogenic factor 5) es una de las proteínas de superficie presentes en esta línea celular y sirve de marcador en las técnicas de identificación ${ }^{9,10}$. El PRDM16 es otra molécula recientemente identificada como regulador transcripcional, decisiva para la evolución a adipocito marrón. El PPAR $\gamma$ (receptor activado por el proliferador de peroxisoma) es un factor de transcripción característico en todos los adipocitos, y la UCP-1 es la proteína desacoplante característica al adipocito marrón ${ }^{9}$. Así, el adipocito marrón tiene como marcadores Myf5, UCP-1 y PPAR $\gamma$, y el adipocito blanco solo presenta este último.

Esta revisión tiene por objetivo exponer los hallazgos sobre los diferentes tipos de adipocitos y su relación con la irisina. Esta hormona representa una promesa en el desarrollo de nuevos tratamientos antiobesidad (Tabla 1).

\section{Tejido adiposo blanco}

EI WAT es el más abundante y es la reserva corporal de energía, que se almacena en forma de triglicéridos. El WAT se localiza en todo el cuerpo y se subdivide a su vez en visceral y subcutáneo. El tejido adiposo visceral (VAT, visceral adipose tissue) está relacionado directamente con el riesgo de desarrollar resistencia a la insulina. Su principal función es la homeostasis energética, pero actualmente se reconoce su capacidad endocrina al participar en la regulación del metabolismo energético y la expresión de citocinas proinflamatorias ${ }^{10}$.

La absorción de lípidos en el tejido adiposo blanco está mediada por la lipoproteína lipasa, unida al endotelio vascular a través de proteoglicanos de sulfato de heparina, con sitios activos ubicados en la superficie luminal de los vasos sanguíneos, lo que permite hidrolizar triglicéridos para ser movilizados y utilizados en el almacenamiento celular ${ }^{11}$. El adipocito blanco presenta una gota lipídica grande, que abarca la mayor parte de su citoplasma, y su núcleo está localizado en la periferia de la célula. Los lípidos se encuentran libres dentro del adipocito y corresponden a depósitos de triglicéridos ${ }^{12}$. El acúmulo excesivo de lípidos en los adipocitos del WAT se asocia con mayor riesgo cardiovascular y metabólico, relacionado con la 


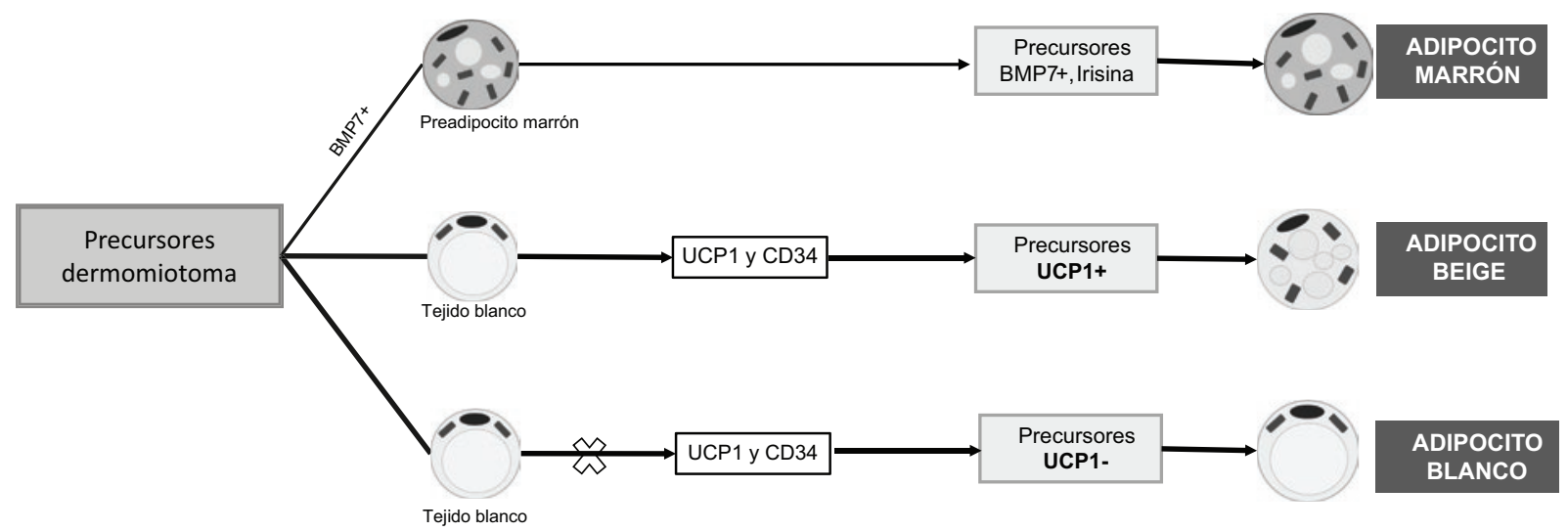

Figura 1. La diferenciación inicia en los dermomiotomas; a partir de la subpoblación de preadipocitos marrones, con marcadores BMP7 e irisina positivos, se originan los adipocitos pardos. Los precursores de los adipocitos blancos que no expresan BMP7 y UCP1 se convierten en adipocitos blancos. Por otra parte, los preadipocitos blancos que expresan UCP1 y algunos otros marcadores (ZIC1 negativos, Cd137 y TMEM26 positivos) se transforman a adipocitos beige.

Tabla 1. Características de los adipocitos blanco, marrón, beige, azul y rosa

\begin{tabular}{|c|c|c|c|c|c|}
\hline & Blanco & Marrón & Beige & Azul & Rosa \\
\hline Localización & $\begin{array}{l}\text { Inguinal, mesentérica } \\
\text { (retroperitoneal, } \\
\text { perigonadal, omental) }\end{array}$ & $\begin{array}{l}\text { Interescapular, perirrenal, } \\
\text { axilar y paravertebral }\end{array}$ & Tejido adiposo blanco & $\begin{array}{l}\text { Hepática, espacio de } \\
\text { Disse, subyacente a } \\
\text { células endoteliales }\end{array}$ & $\begin{array}{l}\text { Glándula } \\
\text { mamaria }\end{array}$ \\
\hline Morfología & $\begin{array}{l}\text { Uniloculares, con } \\
\text { grandes gotas de } \\
\text { lípidos }\end{array}$ & $\begin{array}{l}\text { Multiloculares, con } \\
\text { pequeñas gotas de } \\
\text { lípidos }\end{array}$ & $\begin{array}{l}\text { Vacuolas lipídicas } \\
\text { múltiples }\end{array}$ & $\begin{array}{l}\text { Multilocular, } \\
\text { conteniendo } \\
\text { vitamina A }\end{array}$ & Multilocular \\
\hline Función & $\begin{array}{l}\text { Almacenamiento de } \\
\text { energía en forma de } \\
\text { triglicéridos }\end{array}$ & Producción de calor & Termogénesis adaptativa & $\begin{array}{l}\text { Homeostasis de } \\
\text { la matriz extracelular }\end{array}$ & $\begin{array}{l}\text { Secreción } \\
\text { láctea }\end{array}$ \\
\hline $\begin{array}{l}\text { Generación de } \\
\text { mitocondrias }\end{array}$ & + & +++ & Posestimulación ++ & * & * \\
\hline $\begin{array}{l}\text { Vascularización/ } \\
\text { capilares }\end{array}$ & Bajo & Abundante & $\begin{array}{l}\text { La estimulación por frío } \\
\text { aumenta la angiogénesis }\end{array}$ & * & * \\
\hline UCP-1 & Casi indetectable & +++ & $\begin{array}{l}\text { Posestimulación } \\
\text { (abundante) }\end{array}$ & * & * \\
\hline $\begin{array}{l}\text { Receptores } \\
\text { adrenérgicos }\end{array}$ & $\beta_{3}++, \alpha_{2}+$ & $\beta_{3}+++$ & $\beta_{3} / \alpha_{2}$ & * & * \\
\hline $\begin{array}{l}\text { Relación con } \\
\text { obesidad y } \\
\text { resistencia a la } \\
\text { insulina }\end{array}$ & Efecto positivo & Negativa & Negativa & $\begin{array}{l}\text { Efecto negativo en la } \\
\text { homeostasis de lípidos y } \\
\text { resistencia a la insulina }\end{array}$ & * \\
\hline Activadores & Dieta alta en grasas & $\begin{array}{l}\text { Hormonas tiroideas, } \\
\text { tiazolidinedionas, FGF21, } \\
\text { BMP7, BMP8B, péptido } \\
\text { natriurético }\end{array}$ & $\begin{array}{l}\text { Irisina, FGF21, } \\
\text { péptido natriurético, } \\
\text { catecolaminas, (agonistas } \\
\beta) \text { tiazolidinedionas }\end{array}$ & $\begin{array}{l}\text { Lesión de células } \\
\text { parenquimatosas, } \\
\text { estrés } \\
\text { oxidativo }\end{array}$ & * \\
\hline
\end{tabular}

BMP: proténa morfogenética de hueso; FGF21: factor fibroblástico 21; UCP-1: proteína de desacoplamiento 1.

${ }^{*}$ Datos controversiales o desconocidos.

liberación de citocinas proinflamatorias con actividad secretora, tales como TNF- $\alpha$, interleucina 8 y proteína C reactiva ${ }^{13}$.
El tejido adiposo visceral, con respecto al WAT subcutáneo, presenta mayor infiltración de células inflamatorias e inmunitarias, que son fuente de 
diferentes citocinas inflamatorias ${ }^{12}$. El exceso de VAT se asocia a un mayor riesgo cardiovascular, aterosclerosis, hipertensión y diabetes mellitus tipo 2 (DM) que el tejido adiposo subcutáne $0^{13}$. Otra diferencia del WAT visceral es su gran sensibilidad a los estímulos lipolíticos que incrementan los ácidos grasos libres en la circulación portal y que conducen a una mayor resistencia a la insulina. En comparación con el WAT subcutáneo, los adipocitos viscerales presentan un incremento de los receptores para glucocorticoides y un aumento de la actividad de la lipoproteína lipasa $^{12}$.

\section{Tejido adiposo marrón o pardo}

El tejido adiposo marrón, en contraste con el WAT, está formado por adipocitos de menor tamaño conformados por múltiples y pequeñas gotas lipídicas (multilocular). Los adipocitos de la grasa marrón se originan a partir de células precursoras del mesodermo embrionario. Histológicamente, otras características que distinguen al tejido marrón del blanco son la vascularización y la inervación. Funcionalmente, la característica principal del BAT es la lipólisis y la liberación de energía. Así, los individuos con depósitos menores de BAT presentan una mayor susceptibilidad a acumular WAT y aumentar su peso corporal, y un mayor riesgo de enfermedades cardiovasculares ${ }^{14}$.

La oxidación de los lípidos en el tejido marrón para producir calor se debe a la presencia de una gran cantidad de mitocondrias y de la proteína de desacoplamiento (UCP1). Esta proteína favorece la liberación de energía ${ }^{15}$. Dicha función es especialmente importante en el recién nacido para impedir la hipotermia. El BAT es más abundante en el recién nacido, y se distribuye en las regiones interescapular, cervical, supraclavicular, paravertebral, paraaórtica y suprarrenal15,16. Conforme avanza la edad, el BAT pierde mitocondrias y puede transformarse en grasa blanca, y se encuentra en menor cantidad ${ }^{16}$.

En el sistema nervioso central se producen catecolaminas y orexina, consideradas potentes activadores del desarrollo de grasa marrón y de la función termogénica ${ }^{14}$. Los macrófagos activados que residen en el tejido adiposo secretan también algunas moléculas que estimulan la termogénesis ${ }^{16}$.

El balance entre los tejidos adiposos blanco y marrón puede modificarse en respuesta a distintos factores, como el frío, el calor, la obesidad y la edad; de tal forma que los individuos con menos BAT presentan más susceptibilidad para acumular tejido adiposo blanco y mayor riesgo de enfermedades metabólicas y cardiovasculares ${ }^{17}$.

\section{Tejido adiposo beige}

Se ha observado que en el WAT pueden existir adipocitos con características de adipocito marrón, lo que ha llevado al concepto de adipocito beige o brite (este último término derivado de las palabras en inglés brown and White). Estos adipocitos tienen similitud con los adipocitos marrones y propiedades termogénicas.

El adipocito beige se descubrió en un experimento con animales en el que se observó que cuando estos se exponían crónicamente al frío trasformaban su grasa blanca en marrón, sugiriendo la necesidad de generar energía térmica. En otro experimento, también en animales sometidos a una dieta hipercalórica, se demostró que algunos depósitos de BAT se convertían en grasa blanca, lo que sugirió que el BAT tenía la capacidad de adaptarse para almacenar energía ${ }^{14,17}$.

Los experimentos anteriores plantearon por primera vez el proceso de transdiferenciación de los adipocitos, esto es, que pueden existir células precursoras de los adipocitos blancos y, según el estímulo a que se les someta, pueden transformarse en adipocitos beige (Fig. 1). La presencia de adipocitos beige en el seno del WAT se ha demostrado en distintas especies y se encuentra bajo el control de diferentes factores, como la exposición crónica al frío, los agonistas adrenérgicos y los agonistas del PPAR $\gamma$, entre los que se encuentran las tiazolidinedionas ${ }^{18,19}$.

Los mecanismos precisos de la conversión del adipocito blanco a beige no se han identificado. Aunque su estructura y función son similares, el adipocito beige, presente en islotes dentro del tejido adiposo blanco, no tiene el mismo origen que el tejido marrón convencional. Al respecto existen tres hipótesis: en la primera, el adipocito beige provendría del adipoblasto por una vía celular alternativa; en la segunda, se originaría de una diferenciación alternativa del preadipocito blanco; y la tercera hipótesis y más aceptada es que el adipocito beige se origina a partir de los adipocitos blancos maduros por una diferenciación directa (transdiferenciación) ${ }^{20}$.

La producción de trifosfato de adenosina (ATP) en el adipocito beige es reemplazada por la generación de calor. Es decir, la oxidación de las grasas y de los hidratos de carbono, que al final de la cadena respiratoria conduce normalmente a la formación ATP, provoca en su lugar la liberación de calor. A esta 


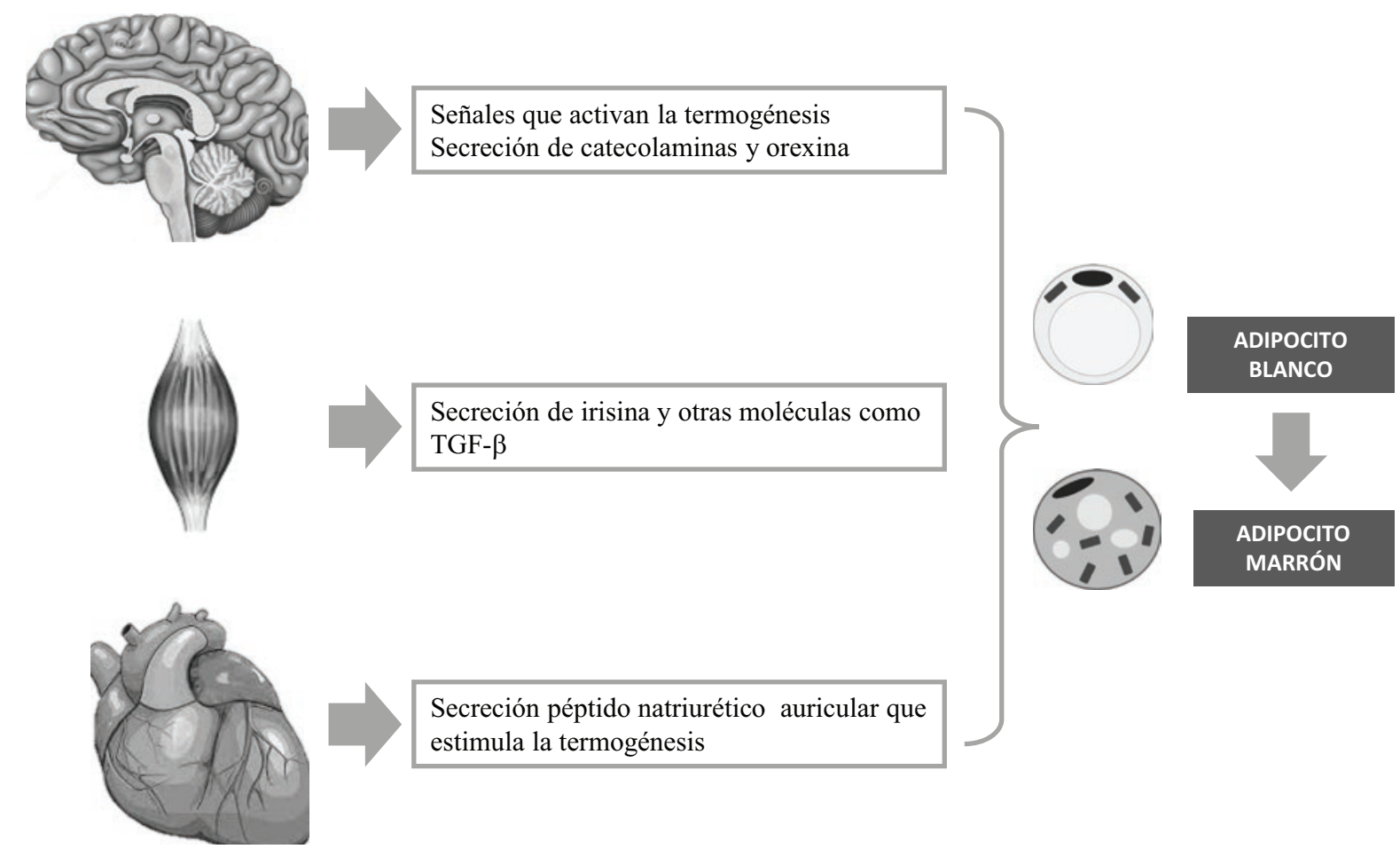

Figura 2. Diferencias moleculares determinan la transformación del adipocito blanco a pardo y, consecuentemente, el aumento de la termogénesis. En el sistema nervioso se producen catecolaminas y orexina, pero existen además otras moléculas secretadas en otros tejidos y órganos, como el músculo esquelético, el corazón y el hígado, que intervienen en la transdiferenciación. TGF- $\beta$ : factor de crecimiento transformante beta.

disociación de la producción de ATP se la llama «desacoplamiento» y sucede gracias a una proteína denominada termogenina, o más comúnmente UCP-I. Esta proteína desacoplante, localizada en la membrana mitocondrial interna, impide el transporte membranal de protones y dificulta la función de la ATP sintetasa; de esta forma, la energía acumulada por la transferencia de electrones en los complejos de la cadena respiratoria se libera en forma de calor ${ }^{9,17}$.

Una hormona recientemente identificada, la irisina, sintetizada por el músculo, tiene la capacidad de conferir a los depósitos de grasa blanca ciertas propiedades características de la grasa marrón, específicamente la estimulación de la termogénesis. Otras moléculas, como el factor de crecimiento transformador beta y el péptido natriurético, estimulan también la termogénesis y el desarrollo de BAT por medio del pardeamiento de la grasa blanca. Desde el hígado, el factor de crecimiento fibroblástico 21 también es un mediador de este proceso de transdiferenciación (Fig. 2).

\section{Adipocito rosa}

El adipocito rosa recibe esta denominación por la coloración rosa que toma con la tinción de hematoxilina-eosina ${ }^{7}$. El adipocito rosa se identificó en el tejido adiposo subcutáneo de ratones en etapa de gestación y de lactancia. El adipocito rosa corresponde a células alveolares de la glándula mamaria, que almacenan gran cantidad de lípidos y su principal función es la producción y secreción láctea ${ }^{21,22}$.

Durante el embarazo y la lactancia, los depósitos de tejido adiposo subcutáneo son sustituidos por células alveolares con abundantes lípidos. Las células rosas se originan probablemente de un proceso de transdiferenciación a partir del tejido adiposo blanco subcutáneo, y bajo control hormonal adquieren progresivamente características epiteliales. El proceso de transdiferenciación probablemente es reversible en la glándula mamaria del ratón; es decir, el epitelio puede diferenciarse a adipocito y el adipocito a células epiteliales.

En la mujer no se ha identificado aún este proceso, pero el conocimiento de los mecanismos de transdiferenciación podría ayudar a comprender aspectos relacionados con la aparición de tumores mamarios ${ }^{22}$. Otra observación interesante es que el adipocito rosa, además, expresa adipocinas como la leptina y la perilipina $A$, las cuales probablemente participan en la prevención de la obesidad en las crías de ratón ${ }^{21}$. 


\section{Adipocito azul}

En el hígado se encuentran células con aspecto estrellado que característicamente almacenan lípidos, conocidas como lipocitos o células estrelladas. Estas células también reciben el nombre de adipocito azul, por la coloración que toman sus vesículas lipídicas por autofluorescencia ${ }^{23}$. Se ubican en el espacio perisinusoidal entre los hepatocitos y las células endoteliales. El adipocito azul tiene un papel fundamental en la homeostasis de los retinoides y la remodelación de la matriz extracelular ${ }^{24}$. El adipocito azul, en su estado quiescente o inactivo, presenta una gran cantidad de vesículas de grasa citoplasmática que constituyen el principal almacén de vitamina A en el cuerpo.

Como consecuencia de diversos estímulos, como la inflamación crónica, el consumo de alcohol o infecciones, entre otros, los adipocitos azules pasan a un segundo estado de activación, perdiendo sus vesículas grasas y sufriendo un proceso de transdiferenciación a células proliferativas y miofibroblastos. Durante esta etapa de activación, el adipocito azul expresa distintas adipocinas, como leptina, resistina, quimerina, adiponectina e interleucinas, entre otras, que afectan el metabolismo lipídico y aumentan la resistencia a la insulina y la respuesta inmunitaria. Luego sobreviene la fibrinogénesis y eventualmente la fibrosis hepática ${ }^{25}$.

Es tema de controversia si la célula azul es parte del tejido adiposo; sin embargo, por algunas características que presenta, tales como almacenamiento de la grasa, producción de adipocinas y su participación en el metabolismo lipídico, parece adecuada la designación de adipocito azul.

\section{Irisina}

La irisina es una molécula recientemente identificada y ha llamado la atención porque es secretada por el músculo esquelético y puede ser una señal de comunicación directa con el tejido adiposo. Es por esta característica que ha recibido su nombre, similar al de la diosa griega Iris, que comunicaba a los dioses del Olimpo con los mortales.

En 2012, Boström, et al. ${ }^{26}$ identificaron por primera vez la irisina, observando en modelos múridos que su aumento incrementaba el gasto energético, controlaba el aumento de peso inducido por dieta y mejoraba la resistencia a la insulina.
Un hecho trascendente es que la irisina tiene la capacidad de transdiferenciar el adipocito blanco a beige, al promover la expresión de UCP-1. La irisina transforma la grasa blanca activando la p38 de la proteína cinasa activada por mitógenos (MAPK). La activación de MAPKp38 es esencial para la expresión de PGC1 $\alpha$ (coactivador $\alpha$ del PPAR $\gamma)^{27,28}$. El proceso de transdiferenciación, aparentemente, no se presenta en todos los adipocitos. En modelos experimentales se ha observado que los adipocitos del VAT no generan p38 al estimularlos con irisina, lo que sugiere que en este tejido visceral no se presenta transdiferenciación; no obstante, la irisina, por otros mecanismos aún no conocidos por completo, provoca una disminución de la producción de citocinas proinflamatorias ${ }^{29}$.

El ejercicio físico es un estímulo para el PGC $\alpha$, que a su vez estimula la expresión de FNDC5 (fibronectina tipo III proteína 5), a partir de la cual se libera la iri$\operatorname{sina}^{28}$. Una vez secretada, se distribuye por el torrente sanguíneo y actúa en distintas estructuras, como el tejido adiposo, el músculo cardíaco, el hígado, el cerebro, el hueso, el páncreas, el riñón y el ovario. Así, uno de los beneficios del ejercicio es la liberación de miosinas, dentro de las que se encuentra la irisina, que a su vez tiene como efectos el aumento del gasto energético y la oxidación de la grasa. Diferentes estudios clínicos han mostrado que se presenta un incremento de la concentración de irisina tras el ejercicio $^{30}$. Los individuos con mayor actividad física presentan un incremento en la concentración circulante de irisina y una ligera disminución de la resistencia a la insulina, en comparación con los individuos físicamente inactivos ${ }^{27,30}$.

La irisina mejora la sensibilidad a la insulina al aumentar la respuesta de su receptor en el músculo, favorece el metabolismo hepático de la glucosa y los lípidos, y promueve la función de las células $\beta$ del páncreas $^{31}$. En estudios clínicos se ha observado una asociación entre la disminución de la irisina circulante y la resistencia a la insulina ${ }^{32}$. Otros estudios han mostrado también una reducción de la concentración de irisina en pacientes con DM, y en otros se ha encontrado una correlación negativa con la glucosa en ayuno ${ }^{32,33}$. De igual forma, se ha observado una disminución significativa de la irisina en pacientes con DM de reciente diagnóstico ${ }^{34}$.

En las mujeres embarazadas, la irisina se secreta tambien en la placenta y su concentración aumenta durante la gestación. Los estudios en mujeres con DM gestacional son escasos y con datos controversiales, pero se ha señalado que la irisina se encuentra 
disminuida en comparación con las mujeres embarazadas sanas y se ha propuesto como marcador para la DM gestacional ${ }^{35}$.

Otro aspecto de esta hormona es su relación con la presión arterial. En modelos animales, la administración periférica de irisina disminuye la vasoconstricción, estimulando la producción de óxido nítrico y activando la vía de $\mathrm{AMPK}^{36}$. En los humanos, los estudios han mostrado una correlación negativa entre la irisina y la presión arterial (sistólica y diastólica) en mujeres con preeclampsia ${ }^{36}$. En esta área hacen falta más estudios para aclarar la relación de la irisina con la hipertensión arterial.

El efecto de la irisina sobre el peso corporal observado en ratones también se ha encontrado en estudios clínicos. En pacientes con obesidad, la irisina se encuentra disminuida y se asocia negativamente con la circunferencia de la cintura ${ }^{32}$. En pacientes con apnea obstructiva del sueño, de manera independiente del índice de masa corporal (IMC), la irisina se asocia con la apnea ${ }^{37}$, por lo que se ha propuesto que la irisina podría ser una molécula mensajera entre un eje formado por los tejidos muscular, adiposo y cerebral ${ }^{37}$. Aún más, otras investigaciones en modelos experimentales han mostrado que la irisina influye en la neurogénesis del hipocampo y en la diferenciación neural ${ }^{37}$.

La información sobre el tema sugiere que la irisina presenta numerosos beneficios potenciales sobre el peso, el metabolismo y el riesgo cardiovascular. No obstante, de manera paradójica, se ha observado en algunos estudios que la concentración de irisina en pacientes con obesidad se asocia directamente con algunos índices de adiposidad, como el IMC, la circunferencia de la cintura, la cantidad total de grasa y la resistencia a la insulina ${ }^{33,38,39}$. Al respecto, se han señalado dos hipótesis. La primera es que, después del músculo, el tejido adiposo es el segundo sitio de producción de irisina, de tal forma que la tasa de secreción de irisina en músculo/tejido adiposo podría variar en situaciones como la obesidad ${ }^{40}$. Otra hipótesis para explicar la discrepancia de los resultados entre personas delgadas y con obesidad es que la concentración de irisina en estas últimas podría ser un mecanismo compensador para aumentar el gasto de energía, mejorar la sensibilidad a la insulina y mantener la homeostasis metabólica ${ }^{41}$. En un estudio se describió un efecto positivo de la irisina sobre la regeneración de las células $\beta$ pancreáticas a través de la acción de la betatrofina, lo que podría explicar el aumento de la resistencia a la insulina en pacientes no diabéticos con obesidad ${ }^{42}$. De manera similar, en mujeres con obesidad mórbida se ha observado un incremento de la concentración de irisina, con una asociación positiva entre su concentración y el IMC ${ }^{43}$. La resistencia a la irisina en pacientes con obesidad, entonces, probablemente corresponde a un fenómeno similar al de la resistencia a la leptina y la insulina ${ }^{40,44}$.

Las acciones de la irisina en la regulación del metabolismo energético, el aumento de la termogénesis y la sensibilidad a la insulina podrían incidir sobre la salud y las enfermedades metabólicas.

\section{Conclusión}

En resumen, el tejido adiposo presenta aún muchos secretos que es necesario esclarecer, así como difundir los conocimientos acumulados en los años recientes. Una de las principales razones es porque el exceso de este tejido adiposo, la obesidad, representa un problema prioritario de salud.

La irisina es un puente de comunicación entre los tejidos muscular y adiposo, y su acción es notable en la transdiferenciación del adipocito blanco al beige, incrementando su capacidad termogénica. Es decir, en términos sencillos, la irisina puede transformar la grasa «mala» en grasa «buena». Sin embargo, es necesario aclarar algunos aspectos sobre su acción y las diferencias que se presentan entre las distintas condiciones clínicas.

\section{Conflicto de intereses}

Los autores declaran que no tienen ningún conflicto de intereses.

\section{Financiamiento}

Los autores declaran que el presente trabajo no recibió ningún financiamiento.

\section{Bibliografía}

1. Carobbio S, Pellegrinelli V, Vidal-Puig A. Adipose tissue function and expandability as determinants of lipotoxicity and the metabolic syndrome. Adv Exp Med Biol. 2017;960:161-96.

2. Kuo LE, Czarnecka M, Kitlinska JB, Tilan JU, Kvetnansky R, Zukowska Z. Chronic stress, combined with a high-fat/high-sugar diet, shifts sympathetic signaling toward neuropeptide $Y$ and leads to obesity and the metabolic syndrome. Ann N Y Acad Sci. 2008;1148:232-7.

3. Gómez-Hernández A, Perdomo L, Escribano O, Benito M. Papel del tejido adiposo blanco en las complicaciones vasculares asociadas a la obesidad. Clin Invest Arterioscl. 2013;25:27-35.

4. Lima-Martínez M, lacobellis G. Grasa epicárdica: una nueva herramienta para la evaluación del riesgo cardiometabólico. Hipertens Riesgo Vasc. 2011;28:63-8. 


\section{B. Corina-Sosa, et al.: Tejido adiposo e irisina}

5. Fernández Muñoz M, Basurto Acevedo L, Córdova Pérez N, Vázquez Martínez A, Tepach Gutiérrez N, Vega García S, et al. Epicardial adipose tissue is associated with visceral fat, metabolic syndrome, and insulin resistance in menopausal women. Rev Esp Cardiol (Eng ed). 2014:67:436-41.

6. lacobellis G, Malavazos A, Corsi M. Epicardial fat: from the biomolecula aspects to the clinical practice. Int J Biochem Cell Biol. 2011;43:1651-4

7. Cinti S. Pink adipocytes. Trends Endocrinol Metabol. 2018;29:651-6.

8. Sánchez JC, Romero CR, Muñoz LV, Rivera RA. El órgano adiposo, un arcoíris de regulación metabólica y endocrina. Rev Cuba Endocrinol. 2016;27:105-9.

9. Wahli W, Constantin N. Les secrets du tissu adipeux. À quoi sert la graisse corporelle? Est-elle seulement néfaste? Etat des lieux. De Boeck Supérieur SA. Bruselas, Bélgica; 2014.

10. Sidossis L, Kajimura S. Brown and beige fat in humans: thermogenic adipocytes that control energy and glucose homeostasis. J Clin Invest. 2015;125:478-86.

11. Laforest S, Labrecque J, Michaud A, Cianflone K, Tchernof A. Adipocyte size as a determinant of metabolic disease and adipose tissue dysfunction. Crit Rev Clin Lab Sci. 2015;52:301-13.

12. Yillmaz H, Cakmak M, Darcin T, Inan O, Sahiner E, Demir C, et al Circulating irisin levels reflect visceral adiposity in non-diabetic patients undergoing hemodialysis. Renal Failure. 2016;38:914-9.

13. Lee M, Wu Y, Fried S. Adipose tissue remodeling in pathophysiology of obesity.Curr Opin Clin Nutr Metab Care. 2010;13:371-6.

14. Park A. Distinction of white, beige and brown adipocytes derived from mesenchymal stem cells. World J Stem Cells. 2014;6:33.

15. Jensen MD. Adipose tissue and fatty acid metabolism in humans. J R Soc Med. 2002;95:3-7.

16. Jaworski K, Sarkadi-Nagy E, Duncan R, Ahmadian M, Sul H. Regulation of triglyceride metabolism. IV. Hormonal regulation of lipolysis in adipose tissue. Am J Physiol Gastrointest Liver Physiol. 2007;293:G1-4.

17. Lidell $M$, Betz $M$, Enerbäck S. Brown adipose tissue and its therapeutic potential. J Intern Med. 2014;276:364-77.

18. García M, Pazos P, Lima L, Diéguez C. Regulation of energy expenditure and brown/beige thermogenic activity by interleukins: new roles for old actors. Int J Mol Sci. 2018;19:2569.

19. Seale P, Kajimura S, Yang W, Chin S, Rohas L, Uldry M, et al. Transcriptional control of brown fat determination by PRDM16. Cell Metabol. 2007:6:38-54

20. Giordano A, Frontini A, Murano I, Tonello C, Marino MA, Carruba MO et al. Regional-dependent increase of sympathetic innervation in rat white adipose tissue during prolonged fasting. J Histochem Cytochem. 2005;53:679-87.

21. Giordano A, Smorlesi A, Frontini A, Barbatelli G, Cinti S. Mechanisms in endocrinology: white, brown and pink adipocytes: the extraordinary plasticity of the adipose organ. Eur J Endocrinol. 2014;170:159-71.

22. Giordano A, Murano I, Mondini E, Perugini J, Smorlesi A, Severi I, et al. Obese adipocytes show ultrastructural features of stressed cells and die of pyroptosis. J Lipid Res. 2013:54:2423-36.

23. Friedman S. Hepatic stellate cells: protean, multifunctional, and enigmatic cells of the liver. Physiol Rev. 2008;88:125-72.

24. Sarem M, Znaidak R, Macías M, Rey R. Las células estrelladas del hígado: su importancia en condiciones normales y patológicas. Gastroenterol Hepatol. 2006;29:93-101.

25. Weiskirchen R, Tacke F. Cellular and molecular functions of hepatic stellate cells in inflammatory responses and liver immunology. Hepatobiliary Surg Nutr. 2014;3:344-63.

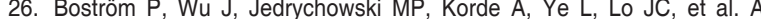
PCG-1 $\alpha$-dependent myokine that drives brown-fat-like development of white fat and thermogenesis. Nature. 2012;48:463-8.

27. Elsen M, Raschke S, Eckel J. Browning of white fat: does irisin play a role in humans? J Endocrinol. 2014;222:R25-R38.

28. Castillo-Quan JI. From white to brown fat through the PGC-1a dependent myokine irisin: implications for diabetes and obesity. Dis Model Mech. 2012;5:293-5.

29. Li H, Zhang Y, Wang F, Donelan W, Zona MC, Li S, et al. Effects of irisin on the differentiation and browning of human visceral white adipocytes. Am J Transl Res. 2019;11:7412-742.

30. Morelli C, Avolio E, Galluccio A, Caparello G, Manes E, Ferraro S, et al. Impact of vigorous-intensity physical activity on body composition parameters, lipid profile markers, and irisin levels in adolescents: a cross-sectional study. Nutrients. 2020;12:1-8.

31. Gizaw M, Anandakumar P, Debela T. A review on the role of irisin in insulin resistance and type 2 diabetes mellitus. J Pharmacopuncture. 2017;20:235-42.

32. Gamas L, Matafome P, Seica R. Irisin and myonectin regulation in the insulin resistant muscle: implications to adipose tissue: muscle crosstalk. J Diab Res. 2015;2015:359159.

33. Martínez-Muñoz IY, Camarillo ES, Garduño JJ. Irisin a novel metabolic biomarker: present knowledge and future directions. Int $\mathrm{J}$ Endocrinol. 2018;2018:7816806

34. Choi YK, Kim MK, Bae KH, Seo HA, Jeong JY, Lee WK, et al. Serum irisin levels in new-onset type 2 diabetes. Diabetes Res Clin Pract. 2013;100:96-101.

35. AL-Ghazali MJ, Ali HA, AL-Rufaie MM. Serum irisin levels as a potential marker for diagnosis of gestational diabetes mellitus. Acta Biomed. 2020; 91:56-63.

36. Maciorkowska M, Musialowska D, Malyszko J. Adropin and irisin in arterial hypertension, diabetes mellitus and chronic kidney disease. Adv Clin Exp Med. 2019:28:1571-5.

37. Huang W, Liu Y, Xu H, Zhu H, Guan J, Yi H, et al. Association of the serum irisin level with obstructive sleep apnea: a body mass index- and physical activity-matched study. Endocrine J. 2020 Mar 18. doi: 10.1507/ endocrj.EJ19-0590. Online ahead of print.

38. Grygiel-Gorniak B, Puszczewicz M. A review on the role of irisin in insulin resistance and type 2 diabetes mellitus. J Pharmacopuncture. 2017;20:235-42.

39. Campolo J, Corradi E, Rizzardi A, Parolini M, Dellanoce C, Di Guglielmo ML, et al. Irisin and markers of metabolic derangement in non-diabetic Caucasian subjects with stage I-II obesity during early aging. PLoS One. 2020;15:e0229152.

40. Moreno M, Moreno-Navarrete JM, Fernández-Real JM. Irisina: ¿transmisor de mensajes del Olimpo? Clin Invest Arterioscl. 2014;26:140-6.

41. Perakakis N, Triantafyllou GA, Fernandez Real JM, Huh JY, Park KH, Seufert J, et al. Physiology and role of irisin in glucose homeostasis. Nat Rev Endocrinol. 2017;13:324-37.

42. Roca-Rivada A, Castelao C, Senin L, Landrove MO, Baltar J, Crujeiras AB, et al. FNDC5/irisin is not only a myokine but also an adipokine. PLoS One. 2013;8:e60563.

43. Pardo M Crujeiras AB, Amil M, Aguera Z, Jiménez-Murcia S, Baños R, et al. Association of irisin with fat mass, resting energy expenditure, and daily activity in conditions of extreme body mass index. Int J Endocrinol. 2014;:2014:857270.

44. Harms M, Seale P. Brown and beige fat: development, function and therapeutic potential. Nat Med. 2013;19:1252-63. 